\title{
The 12Kx8K CCD mosaic camera for the Palomar Transient Factory
}

\author{
Gustavo Rahmer*, Roger Smith, Viswa Velur, David Hale, Nicholas Law, Khanh Bui, Hal Petrie, \\ Richard Dekany
}

Caltech Optical Observatories, MS 105-24, California Institute of Technology, Pasadena, CA 91125

\begin{abstract}
The Palomar Transient Factory is an automated wide-field survey facility dedicated to identifying a wide range of transient phenomena. Typically, a new 7.5 square degree field will be acquired every 90 seconds with $66 \%$ observing efficiency, in g' band when the sky is dark, or in R band when the moon is up. An imaging camera with a $12 \mathrm{Kx} 8 \mathrm{~K}$ mosaic of MIT/LL CCDs, acquired from CFHT, is being repackaged to fit in the prime focus mounting hub of the Palomar 48-inch Oschin Schmidt Telescope. We discuss how we have addressed the broad range of issues presented by this application: faster CCD readout to improve observing efficiency, a new cooling system to fit within the constrained space, a low impact shutter to maintain reliability at the fast observing cadence, a new filter exchange mechanism, and the field flattener needed to correct for focal plane curvature. The most critical issue was the tight focal plane alignment and co-planarity requirements created by the fast beam and coarse plate scale. We built an optical profilometer system to measure CCDs heights and tilts with $1 \mu \mathrm{m}$ RMS accuracy.
\end{abstract}

Keywords: CCD, mosaic, wide-field survey, transients.

\section{INTRODUCTION}

\subsection{The Palomar Transient Factory}

The Palomar Transient Factory (PTF) is a transient search project based at Palomar Observatory. New or varying sources will be searched using an automated 7-square-degree imager on the Samuel Oschin Telescope, a 48-inch Schmidt. Optical transients will be automatically detected and initially classified within the same night, and detailed follow up of interesting events will be immediately scheduled on the Palomar 60 -inch telescope ${ }^{[1]}$ and other facilities. PTF is designed to search for a wide variety of transient sources with characteristic timescales ranging from minutes to months. The system is currently in development and is expected to achieve first light in late 2008.

PTF is a science collaboration of Caltech, Las Cumbres Observatory Global Telescope Network, Columbia University, Weizmann Institute of Science, Lawrence Berkeley National Laboratory, UC Berkeley \& Infrared Processing and Analysis Center (IPAC).

\subsection{Instrument Overview}

The core of the PTF imager is a $12 \mathrm{Kx} 8 \mathrm{~K}$ mosaic array of twelve $2 \mathrm{Kx} 4 \mathrm{~K}$ MIT/LL CCID20 CCDs and vacuum enclosure forming the front end of the CFH12K camera, which was purchased from the Canada-France-Hawaii Telescope (CFHT), where it had been in use at the telescope prime focus between 1999 and 2003. The CCDs are approximately $3.5 \times 7 \mathrm{~cm}$ each $(15 \mu \mathrm{m}$ pixels $)$ in size and are arranged in a $6 \times 2$ array. Out of the 12 devices, nine are made from standard epitaxial silicon (EPI). The remaining three are made from high-resistivity bulk silicon (Hi-Rho) and are grouped in one corner of the mosaic. The Hi-Rho devices have a higher QE (up to $20 \%$ more) in the red part of the spectrum, and produce less fringing than the EPI devices due to their larger thickness ${ }^{[2]}$.

As reported by $\mathrm{CFHT}^{[2]}$, the detectors have an average readout noise of 5 electrons (with a correlated double sampling time of $1 \mu \mathrm{s}$ per dwell), a dark current of only $\sim 1$ electron per minute per pixel at $-89 \mathrm{deg}$. C, and a peak quantum efficiency of up to $90 \%$ in $\mathrm{R}$ band. The average conversion gain is 1.6 electrons/ADU.

*grahmer@astro.caltech.edu; phone 1626 395-2899

Ground-based and Airborne Instrumentation for Astronomy II, edited by lan S. McLean, Mark M. Casali, Proc. of SPIE Vol. 7014, 70144Y, (2008) · 0277-786X/08/\$18 - doi: 10.1117/12.788086 
The data acquisition system originally consisted of two separate Astronomical Research Cameras Generation II CCD controllers, each reading a bank of six CCDs.

The CFHT camera included a shutter and a filter wheel with space for four filters. The diameter of the whole unit was around 3 feet. The cooling of the camera was provided by an 8-liter liquid nitrogen tank. There was also an Auxiliary Control Electronic module, which served as interface to the shutter, filter wheel and temperature control, in coordination with the CCD controllers ${ }^{[2]}$.

Our goal has been to minimize costs by reusing as much as possible of the original camera, while making only the changes necessary for it to fit within the space and operability constraints of its new host telescope. However many modifications have been unavoidable. The original camera was mounted by its front flange to the prime focus corrector of the CFH Telescope, and had ample space for the large liquid nitrogen reservoir on the back of the camera, whereas the new enclosure will be mounted by its rear surface to a focusing hub just 6 inches behind the focal plane. Not only is the axial space constrained, but the footprint must be reduced since the instrument would otherwise obstruct a substantial fraction of the smaller telescope beam. A much more compact filter mechanism was required and the electronics boxes had to be moved to the telescope exterior. The mass also had to be reduced since the supports and focus mechanism were only designed to carry a photographic plate holder. The original flat window was replaced by a field flattener to compensate for the $3 \mathrm{~m}$ radius curvature for the Schmidt focal surface, and the pixel and data transfer rates had to be doubled to maintain observing efficiency at the faster observing cadence.

The focal plane itself (shown in Fig. 1) remains untouched and has not been disassembled. This includes mounting plate, fanout boards, hermetic connectors and the square forward section of the vacuum enclosure. A Joule-Thompson cooler has been mounted within this volume along with a new thermal path with lower impedance to the detectors.

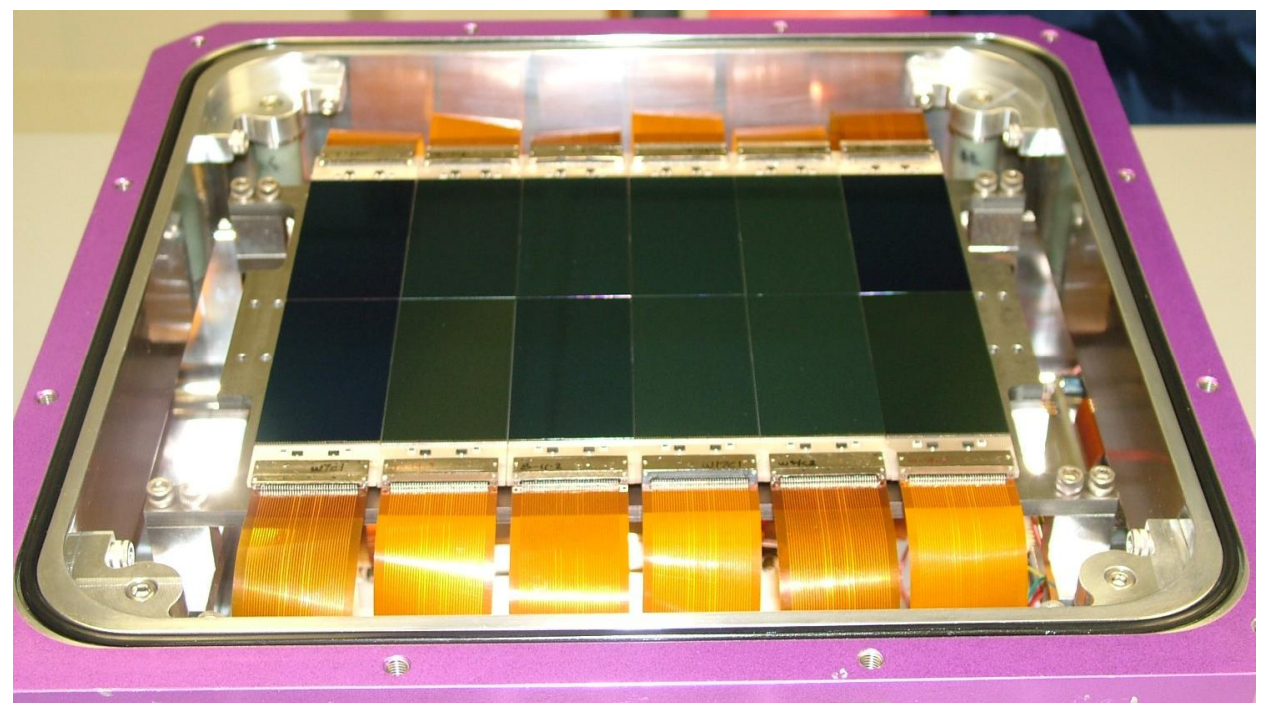

Fig. 1: The Mosaic camera focal plane inside the dewar with front plate and window removed.

Fig. 2 shows a solid model of the new camera setup, as mounted on the telescope spider and focus hub. One of the main objectives of the mechanical redesign effort was to keep the overall footprint of the camera to a minimum. By minimizing the beam obscuration, we achieve the ultimate goal of maximizing the survey efficiency. 


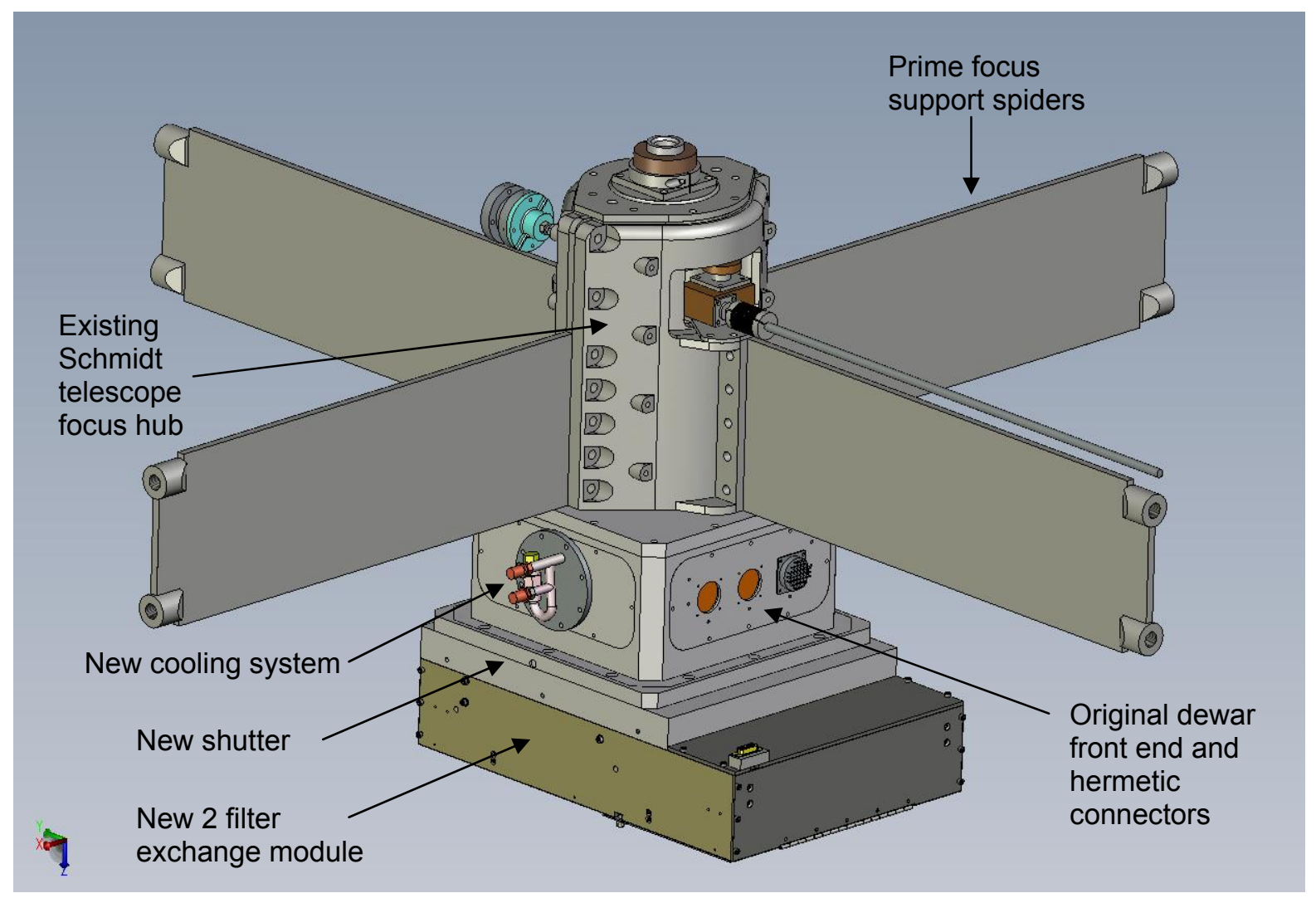

Fig. 2: Solid Model of the mosaic camera mounted on the telescope spiders and focusing hub. Primary mirror is down.

\section{OPTO-MECHANICAL MODIFICATIONS}

\subsection{Field flattener}

The Palomar Schmidt telescope delivers an $\mathrm{f} / 2.45$ beam, which is faster than the $\mathrm{f} / 4.18$ beam delivered by the CFHT at prime focus. An optical design was made to reduce the spot size and maximize the encircled energy in a pixel, specifically in the R and g' bands. As is usually the case for field flatteners, performance was improved when the distance from the rear (flat) surface to the CCD was minimized and the lens thickness was reduced. The reduction in lens thickness is limited by stresses due to atmospheric pressure. We adopted a rectangular shape to minimize the unsupported span. The lens had to be increased in width to allow the supporting frame to stay clear of the flat cables coming out of the CCDs.

The final design of the field flattener resulted in a $216 \mathrm{~mm}$ x $216 \mathrm{~mm}$ plano-convex fused silica lens with $974 \mathrm{~mm}$ radius of curvature, $15 \mathrm{~mm}$ thickness at the center and $4 \mathrm{~mm}$ thickness at the corners. By Finite Elements Analysis, the maximum von Mises stress was determined to be 1000 psi when the planar side is subject to vacuum, which is a conservative design for a fused silica optic. At $3 \mathrm{~mm}$ separation from the CCD surface, $1.5 \mathrm{~mm}$ clearance is provided between the field flattener and the top of the CCD connectors, as confirmed with our optical profilometer. During the optimization of the field flattener design, the position of the Schmidt corrector with respect to the primary mirror was allowed to vary for minimum image size (maximum encircled energy). The new position is approximately 1 inch above the current corrector position, and is in conformance with the original prescription for the telescope.

Spot diagrams for the g' filter are shown in Fig. 3, and the corresponding encircled energy is shown in Fig. 4. The distortion is $0.15 \%$ at the corners of the array relative to a straight grid. The PTF pipeline software will correct for this distortion during the initial data calibration. 


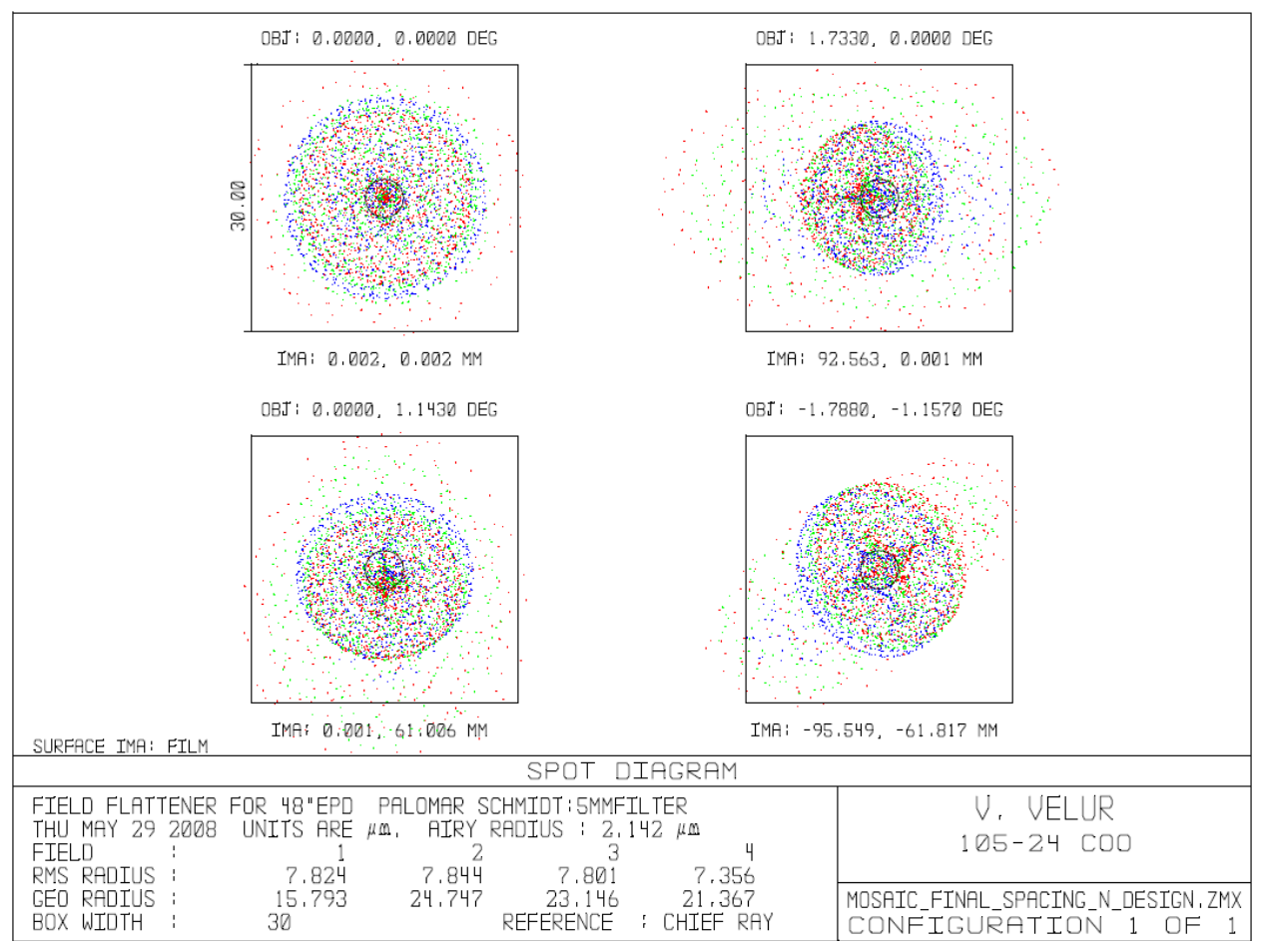

Fig. 3: The spot diagram for the g' filter resulting from the optical design, for four different field positions (the center and three edges). The box width is $30 \mu \mathrm{m}$ ( 2 pixels).

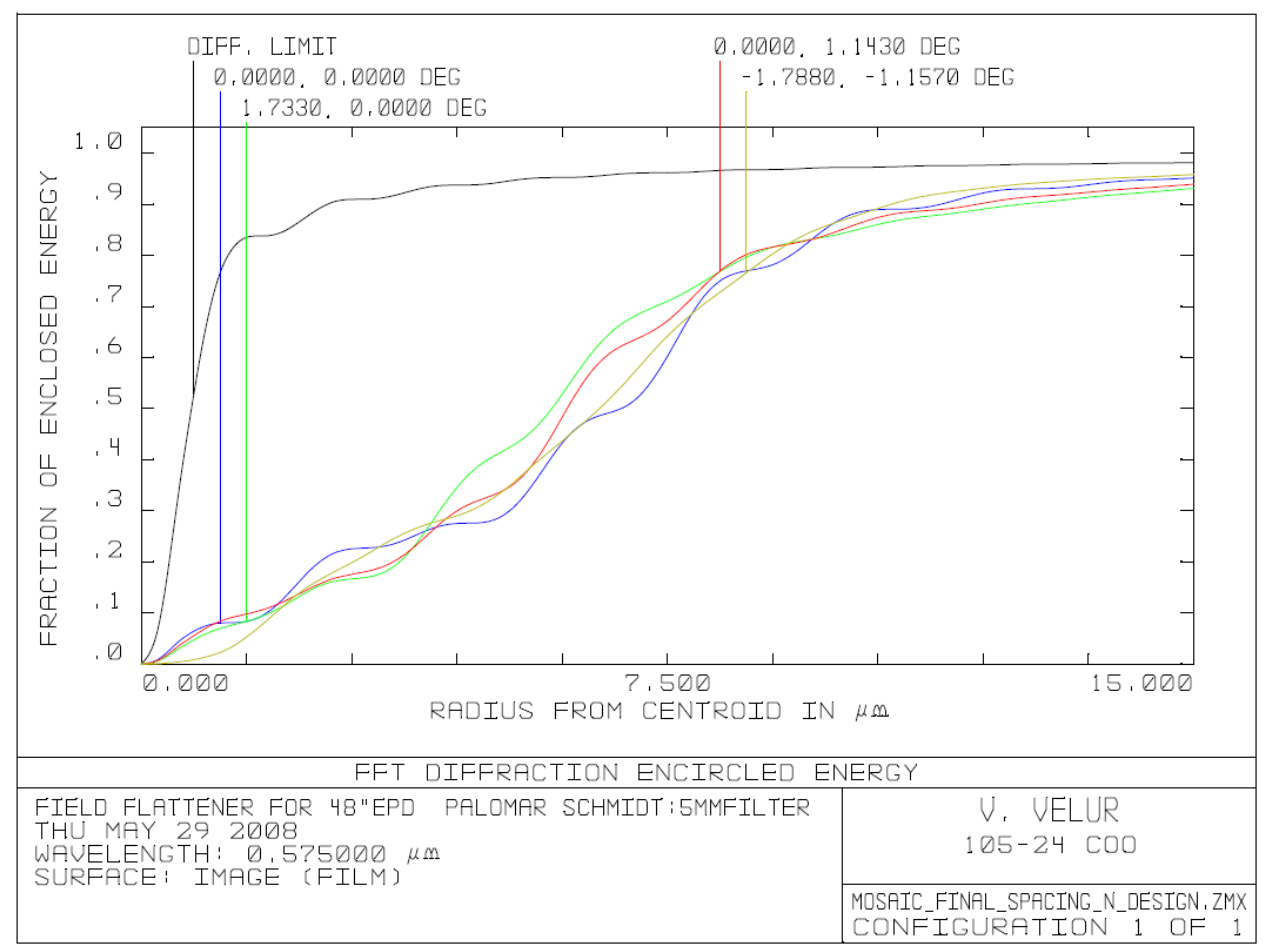

Fig. 4: The encircled energy for the g' filter over the field of view, comparing the performance achieved for the field positions in the previous figure with the diffraction limited case. 


\subsection{Shutter}

The faster exposure cadence of PTF would have resulted in excessive wear on the CFHT shutter, which was cocked by a motor, accelerated by a spring and decelerated rapidly. Additionally we had some concerns that the rapid shutter motion would vibrate the less massive camera and support structure. As the typical exposure time will be 60 seconds, high speed is not required, but precise blade motion is needed to maintain exposure uniformity and timing accuracy of \pm 10 $\mathrm{ms.}^{[3]}$

Scientific Instrumentation Technology built a custom shutter which is larger than their other models but of similar design. It employs dual split blades to avoid beam obscuration by the blades when the shutter is open. Blade motions are precisely controlled, with low acceleration to minimize vibration and wear. A service interval of 500,000 cycles and a lifetime of 2-3 million cycles is promised by the manufacturer. The shutter blades are constructed of carbon fiber to reduce weight and thus vibration. The dual blades are independently controlled, allowing exposure times shorter than the blade transit time. Interfacing to the shutter can be via ASCII commands sent to its RS-232 port, however we intend to synchronize with a TTL signal generated by the CCD controller.

\subsection{Filter exchange mechanism}

A scientific requirement for scheduling flexibility ${ }^{[3]}$ called for having motorized selection between two filters with occasional manual loading (during the day) of different filter pairs. The manual loading will be performed without removing the instrument or filter mechanism from the telescope. The main design challenge was to minimize obscuration of the collimated beam by the housing, while avoiding vignetting at the field edges as might occur if the furthest surface was more than $100 \mathrm{~mm}$ from the CCDs (due to expansion of the $\mathrm{f} / 2.45 \mathrm{beam}$ ). The filters move in different planes, so they can be stored on the same side of the beam. The dimensions of the module, shown in Fig. 5, are $356 \mathrm{~mm} \times 500 \mathrm{~mm} \times 102 \mathrm{~mm}$ with a total weight of $8.63 \mathrm{~kg}$. As seen in Fig. 2, this module does indeed define the beam obscuration produced by the camera, which we have calculated to be $15.3 \%$ on axis, compared to $12 \%$ for the shutter and $10.8 \%$ for the bare CCD dewar. (These figures do not include the telescope spiders which account for another $\sim 4 \%$.)

The two filters usually installed will be g' and R. A custom $250 \mathrm{~mm}$ x $165 \mathrm{~mm}$ SDSS g' filter was ordered from Asahi Corp., Japan. The camera will need a slight focus change when changing filters.

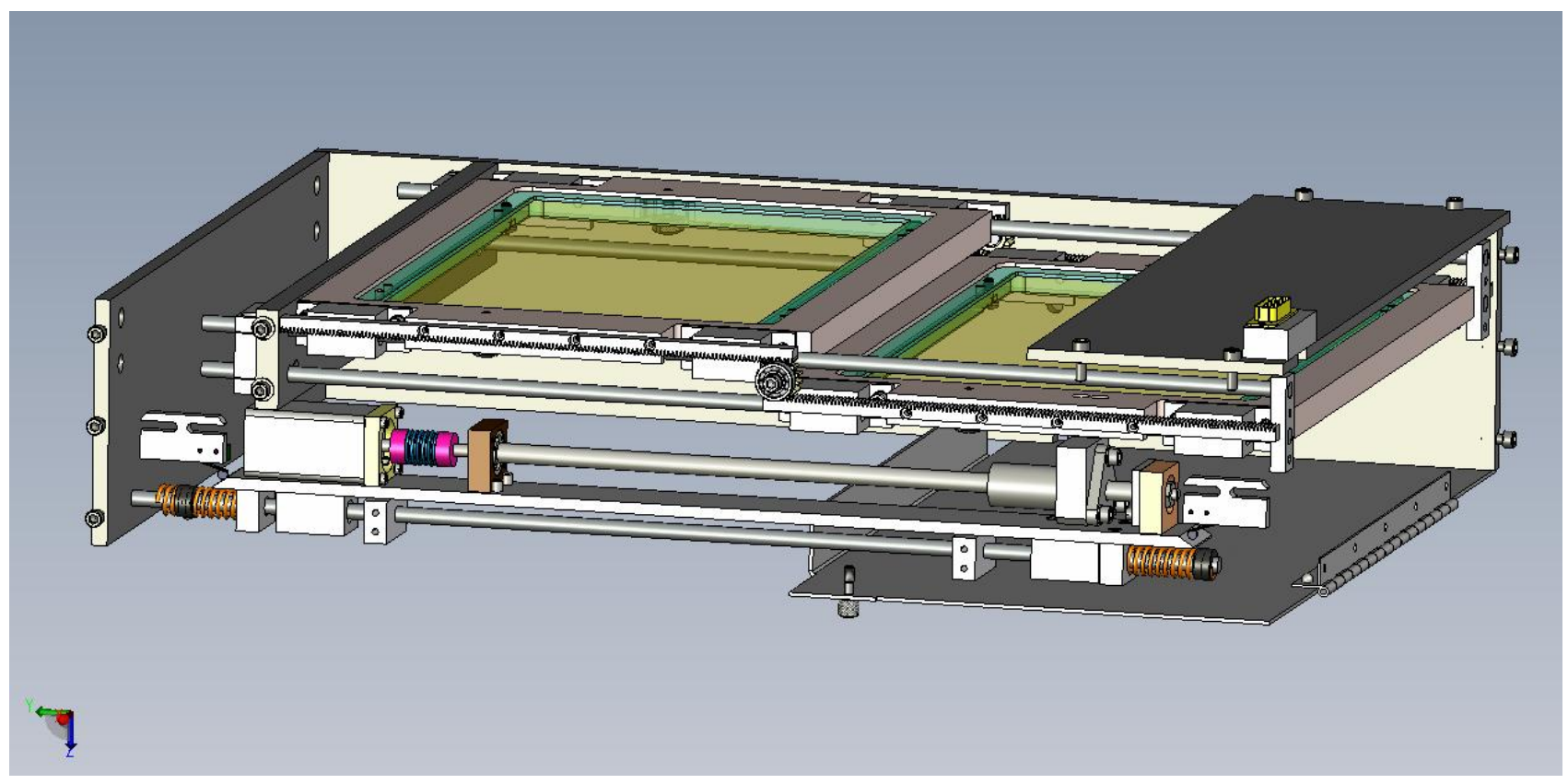

Fig. 5: The new PTF filter exchange module. Two filters in different planes are driven in opposite directions by a single motor and park on one side of the beam to minimize obscuration. The furthest filter surface is $96.5 \mathrm{~mm}$ from the CCDs near the maximum permitted by the angle of the $\mathrm{f} / 2.45$ beam. Light enters from below. 


\section{COOLING SYSTEM}

We chose to replace the original liquid nitrogen dewar with a closed-cycle cooling system, which provides a compact head located close to the CCDs, eliminating nitrogen fills and allowing the dewar to be removed from the telescope without disassembly. A Joule Thompson closed-cycle cooler ("Polycold Compact Cooler", formerly known as Cryotiger) consisting of an expansion chamber with no moving parts was chosen to minimize vibration. Our simulations predict (Fig. 6) that the standard head with PT-30 gas blend will attain a final temperature of $127 \mathrm{~K}$, cooling from ambient to operating temperature $(\sim 175 \mathrm{~K})$ in less than 5 hours. The cooling power, heat capacities and thermal resistances can be predicted with confidence, while the less well defined radiative transfer is based on conservative values for emissivity. The projected radiative load plus conduction is $14 \mathrm{~W}$, so that $8 \mathrm{~W}$ of heater power can be applied while still retaining $8 \mathrm{~W}$ of spare cooling power since the peak cooling power with PT30 refrigerant is $30 \mathrm{~W}$ (at 132K). Provision has been made in the design to include a floating radiation shield on the back and sides of the focal plane to provide additional thermal margin.

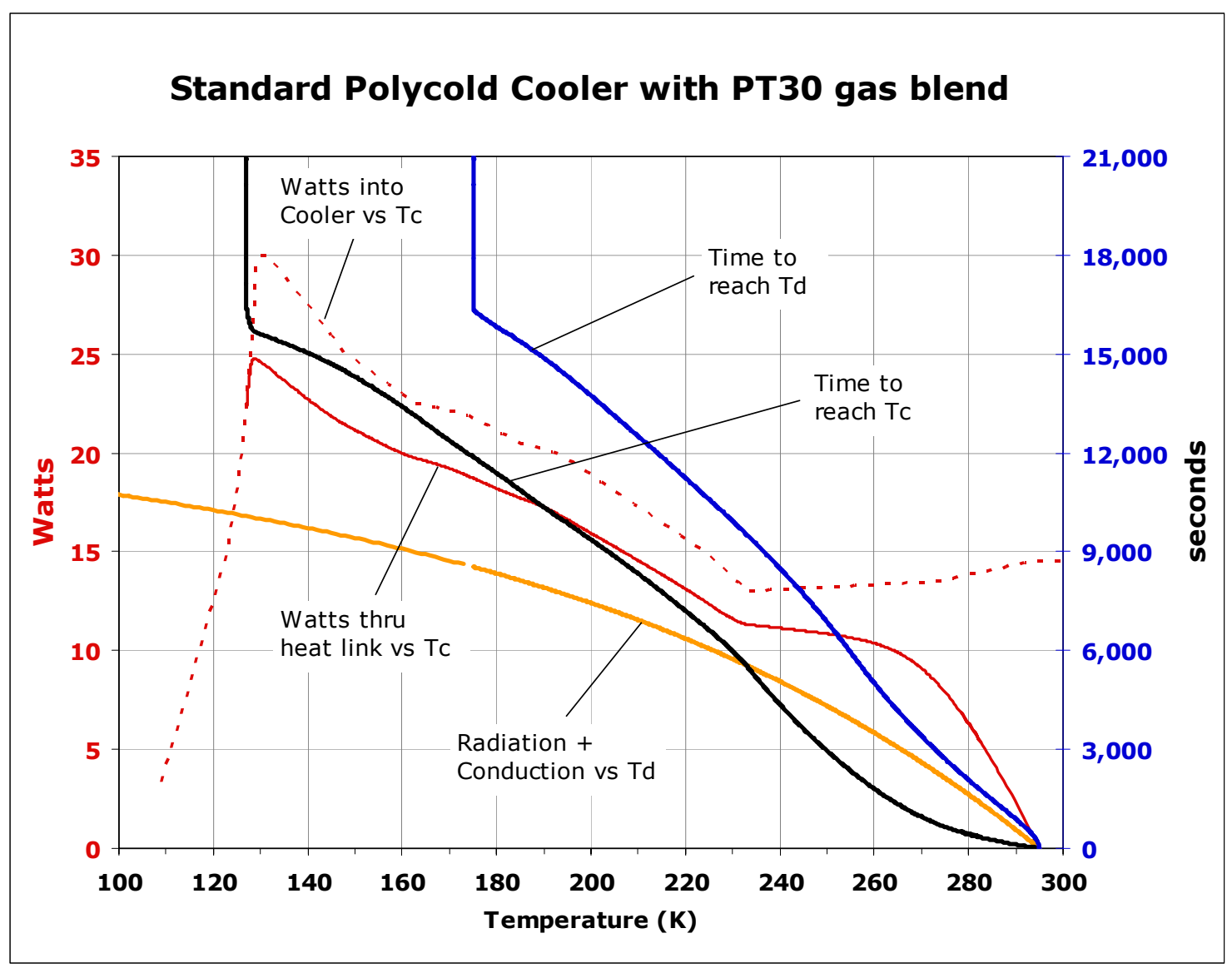

Fig. 6: Time to reach a given temperature cooling from ambient is given for the cold head (Tc) and detector assembly (Td) in seconds on the right axis. The gray curves, read from the left axis, show the power cooling capacity versus cold head temperature (dotted), the actual heat flow into the cold head (fine, solid line) and the combined radiative and conductive flow (thick, solid line). The dominant heat flow is the radiation from window to CCD. Emissivities of both have been assumed to be 1 . 


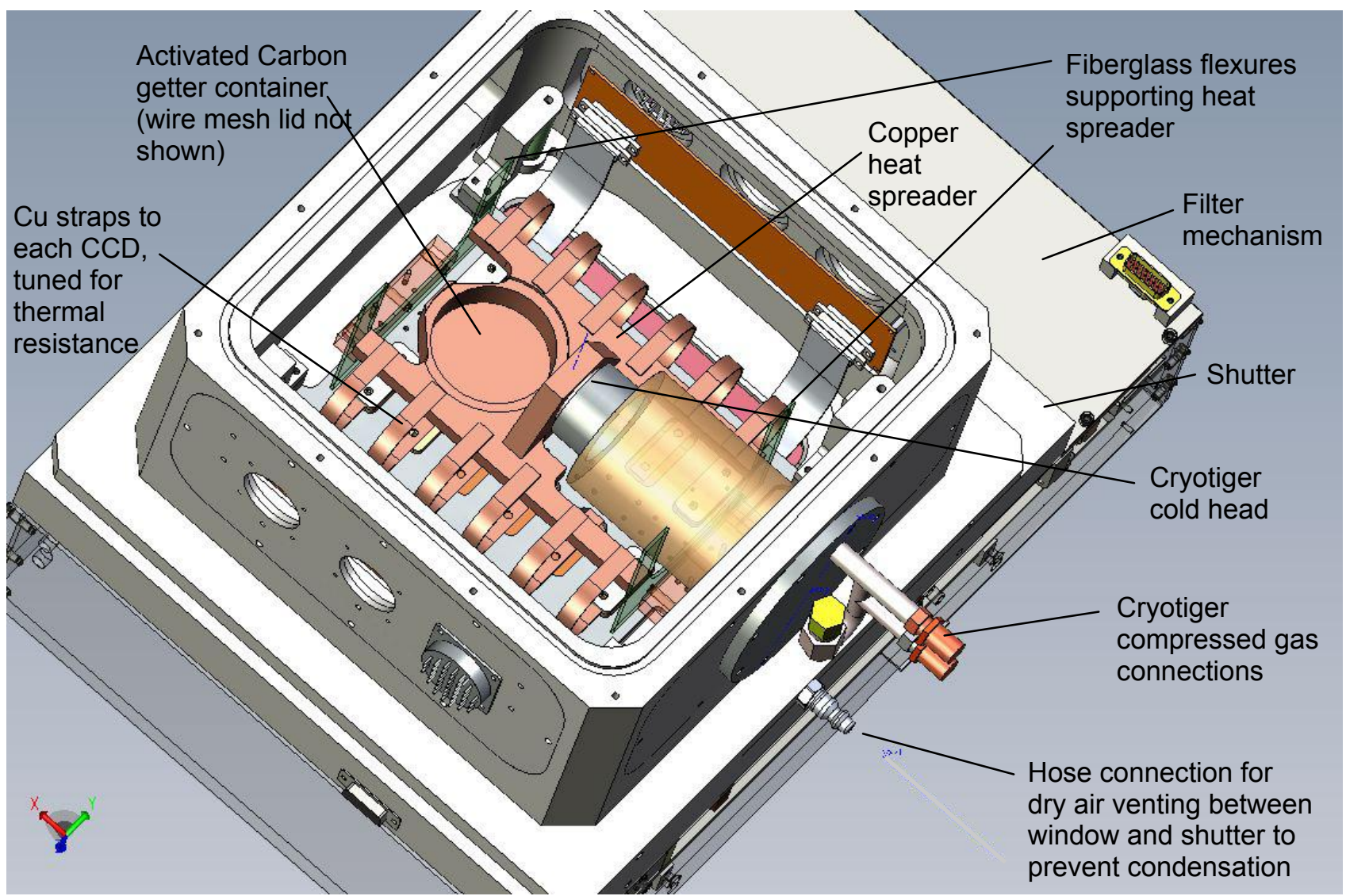

Fig. 7: Solid model showing cryocooler installation through side hatch and thermal path with back cover removed.

An H-shaped copper heat spreader (Fig. 7) has been attached to the cold head to provide a nearly isothermal surface to which the flexible copper straps to the CCDs are attached so that focal plane cooling is uniform. The straps have been shortened and increased in width to allow for the cryocooler not reaching liquid nitrogen temperature. While the heat spreader weighs slightly less than the $1.4 \mathrm{~kg}$ static load limit at the cryocooler tip, the cryocooler is susceptible to fatigue-induced failure due to vibrations. As a result the manufacturer recommends moving the cryocooler head to its original shipping container during transport so that it does not even carry its own weight. However a requirement for disassembly of the dewar for transport and general susceptibility to damage during handling are highly undesirable features, so we have supported the heat spreader and thus the cryocooler with fiberglass sheets which constrain radial motion of the tip while flexing to allow axial motion due to contraction during cooling. According to the manufacturer, a 0.5 " diameter stainless steel tube running along the central axis from the mounting flange to the cold head supplies the structural strength of the cryocooler. The 0.010 " wall thickness is rated to carry $11.4 \mathrm{~kg}$ axial load, which we deem sufficient to support the $1.3 \mathrm{~kg}$ mass of the heat spreader in the axial direction during transport. Thermal contraction will produce up to $125 \mu \mathrm{m}$ radial motion of the cooling head but this is equivalent to the deflection caused by only $450 \mathrm{~g}$ static load at the cooling head, given the compliance of the cantilevered stainless steel tube at the center of the cooler.

Since we were unable to find data quantifying the air pumping efficiency of activated carbon getters at $128 \mathrm{~K}$, we simply included a larger than usual getter container $(\sim 65 \mathrm{ml})$. This is embedded in the heat spreader so that it is at the lowest temperature available. A zeolite getter $(\sim 30 \mathrm{ml})$ at room temperature will have the sole function of adsorbing water vapor. The getter will be located on the interior of a small vacuum hatch so that it can be easily changed without removing the instrument from the telescope. Our expectation is that this will be required only once after initial dry out of the dewar if it has been open for a prolonged period, i.e. only rarely. 


\section{FOCAL PLANE CO-PLANARITY}

The Palomar 48 inch telescope delivers an $\mathrm{f} / 2.45$ beam and thus has less depth of focus compared to the CFH Telescope, which delivers $\mathrm{f} / 4.18$ after the prime focus corrector. Our total delivered image quality goal, 2 arcsec, maps to 2 pixels (at $1 \mathrm{arcsec} / \mathrm{pixel})^{[4]}$, whereas the typical 0.6 arcsec FWHM images on CFHT mapped to 3 pixels at their 0.2 arcsec/pixel plate scale ${ }^{[2]}$. In combination this placed a tighter requirement on flatness of the CCD surfaces. Re-leveling of the array by polishing the shims between the CCDs and their support plate was potentially one of the riskiest and most timeconsuming tasks within the re-engineering effort, and thus something we wished to avoid if possible.

Accurate non-contact profilometry of the mosaic surface was required to determine whether to proceed and would be required again during the re-shimming process so we elected to build a profilometer which could scan an area larger than the array with a resolution and flatness of better than $1 \mu \mathrm{m}$. Most profilometers place the "specimen" on the XY stage and mount the depth sensor on a fixed stand. Instead we placed a non-contact laser displacement sensor (Keyence, Model LK-G82) on the XY stage (Aerotech ALS20000 series) and built a frame to suspend the dewar above the profilometer so that we could scan the mosaic while cold without concern for its mass and size (Fig. 8).

The Keyence sensor, which uses parallax to measure distance rather than best focus, was chosen since it provides the best compromise between accuracy and enough working distance to be able look through the hole in the frame supporting the dewar and through dewar window. It also provides a wide dynamic range allowing the CCD surface and the window and dewar flange to be scanned without moving the sensor in Z. In fact it measures the window front and rear surfaces at the same time as the CCD, providing a crosscheck for any errors in the stage motion. The Keyence sensor is operated in specular rather than diffuse reflection mode, to get sufficient return signal from CCDs with very high QE (and thus low reflectivity) at the red wavelength of the laser. We provided a fine adjustment mechanism to be able to maximize the reflected signal.

The stage has far more precise XY positioning accuracy $( \pm 1 \mu \mathrm{m})$ than required, but was selected to provide reasonably smooth and level motion. The roll specification in the lower stage is most critical since the distance traveled off center by the upper stage multiplies this error. The requirement is only that this error be smooth since $\mathrm{Z}$ errors induced by the stage are calibrated by scanning an optical flat, and subtracted from the CCD height profile.

While the stand holding the mosaic camera is mounted to the base plate for the XY stage to maintain registration, short adjustable legs provide a stiff load path to the concrete floor so that the stage motion does not couple vibrations to the specimen support frame. To date the XY has been stepped between positions with depth measurements being averaged for 1 second at each measurement position while stationary. The motion controller will be upgraded in the future to allow precise synchronization between the scanning and depth measurement so that the stage can move at constant velocity without stopping at each position. This will greatly improve measurement speed and allow multiple scans to be averaged instead of averaging multiple samples at each position, with consequent reduction in susceptibility to long term drift in the sensor readings and thermal expansion effects. 


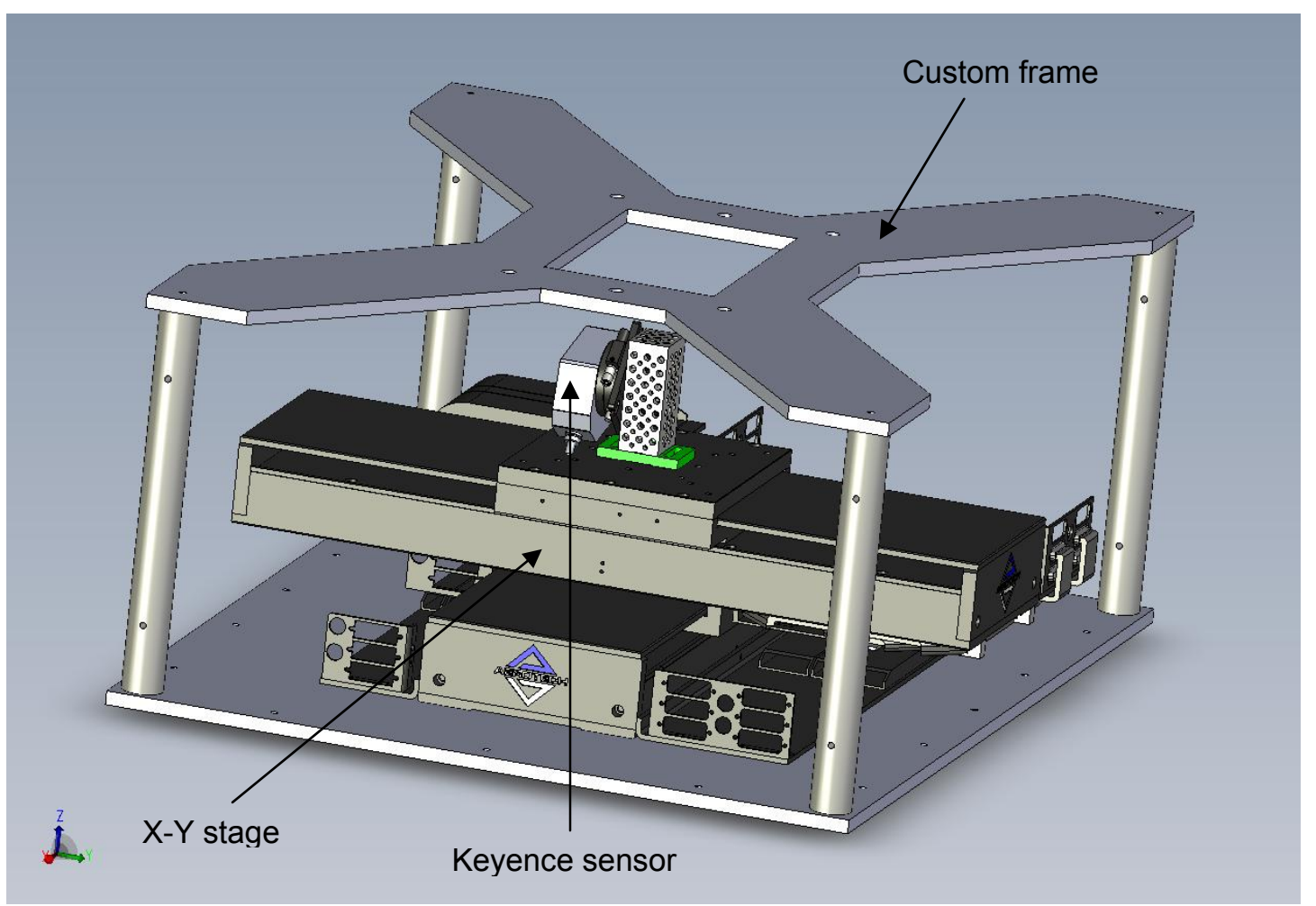

Fig. 8 Profilometer consisting of an XY stage with stiff load path to floor via stiff adjustable legs carries a Keyence laser displacement sensor with working distance of $80 \pm 15 \mathrm{~mm}$ and $0.5 \mu \mathrm{m}$ resolution. It looks upwards to surfaces suspended on a frame which is custom made for each application so that small or large objects can be scanned while looking downwards to minimize accumulation of particles.

The profilometer was calibrated with an optical flat ${ }^{[5]}$ placed on the upper frame. The calibration flat was independently measured to have a sag of $4.4 \mathrm{~nm}$ across its surface and a roughness of $2 \mathrm{~nm}$ RMS in the spatial frequency range from full diameter to $\sim 1 / \mathrm{mm}$. Our own profilometry of the calibration flat showed a peak-to-valley of $10 \mu \mathrm{m}$ with an RMS of $1 \mu \mathrm{m}$, providing a calibration shape and estimate of our measurement precision.

The peak-to-valley deviation from a common plane across the entire instrument CCD mosaic array (Fig. 9) was measured to be $90 \mu \mathrm{m}$ with an RMS of $17 \mu \mathrm{m}$. The white regions in Fig. 9 are areas where we did not get a reading from the laser displacement sensor possibly due to contamination on the dewar window and we speculate that the smaller drop-outs correspond to dust particles on the window surface. 


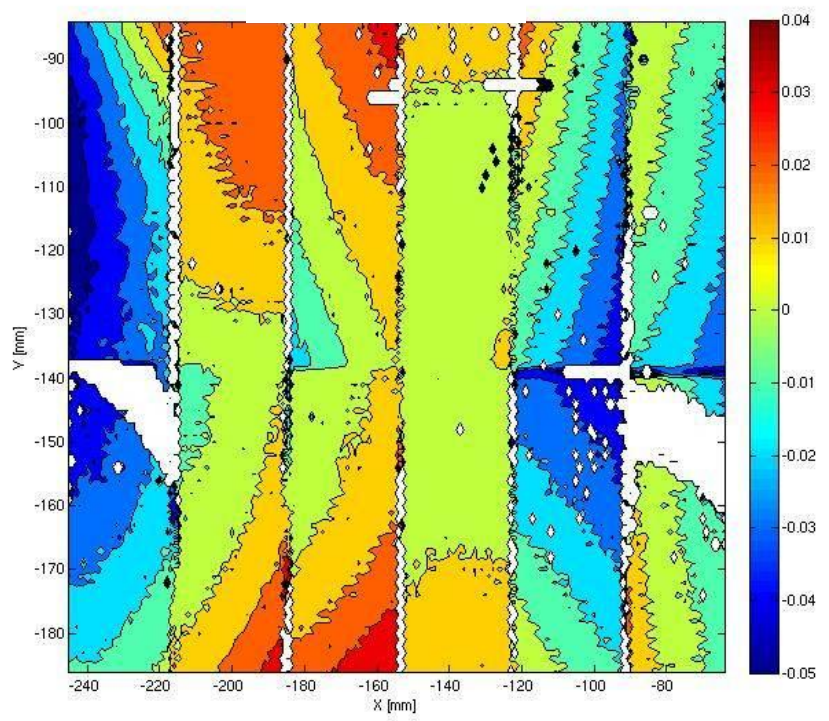

Fig. 9: Contour map of focal plane showing CCD height relative a common plane of best fit (10 $\mu$ m contour intervals). No signal was returned by the profilometer in the white areas.

Individual detectors exhibit peak-to-valley variation between $16 \mu \mathrm{m}$ and $73 \mu \mathrm{m}$, which is dominated by piston and tilt. Fig. 10 depicts the plane of best fit for each detector plotted relative to the plane of best fit for the entire mosaic. These are the focal surface errors which could be compensated by polishing the three contact pads on the molybdenum spacers between each of the CCDs and its molybdenum support plate. The measurements of Fig. 9 and Fig. 10 were made with the dewar cold but were repeated at room temperature. The shape change due to cooling the array was less than $19 \mu \mathrm{m}$ peak-to-valley across the entire array.

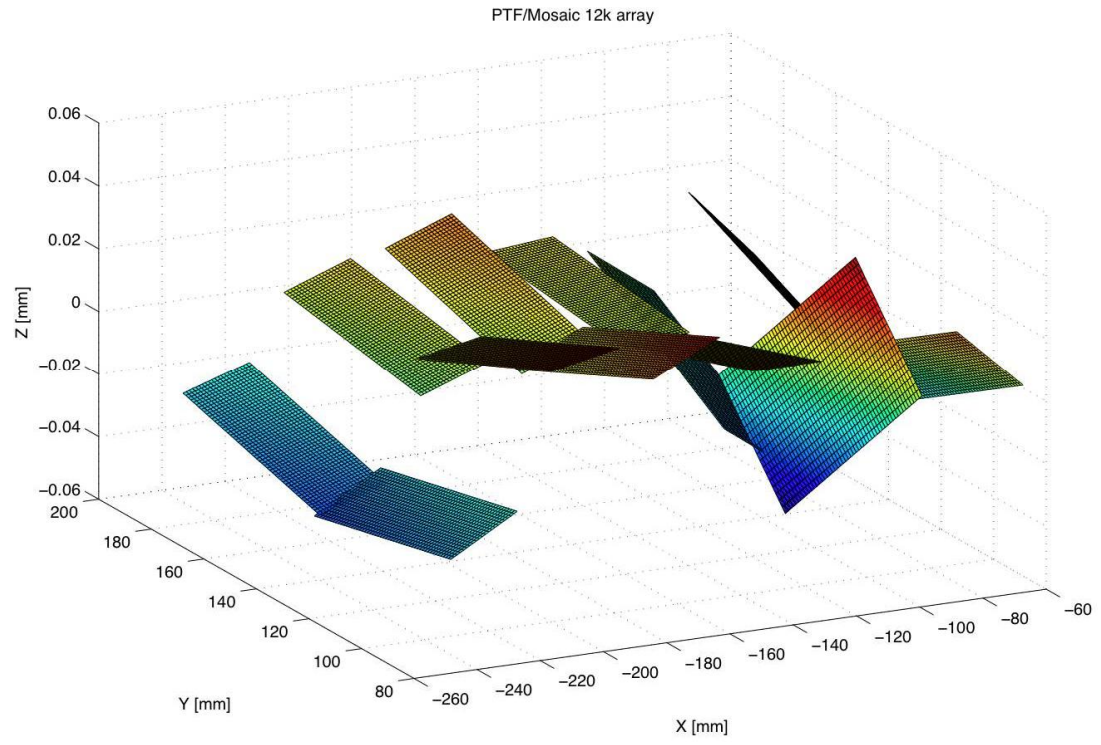

Fig. 10: Plane of best fit for individual CCDs with respect to the common plane for the entire mosaic. These are the piston and tilt errors, which might be removed by polishing the contact pads on the spacers between the CCDs and their support plate 
PTF science requires images no worse than 2 arcsec FWHM (2 pixels) in median Palomar seeing conditions, with $80 \%$ of the flux enclosed in a circle of 2 arcsec radius ${ }^{[4]}$. Fig. 11 shows the distribution of FWHMs produced by the CCD tilt and piston errors measured with the profilometer. On the basis of a detailed image quality error budget calculation, taking into account the variation in optical spot size across the array, we predict that $89 \%$ of the focal plane will deliver image quality better than the required 2.0 arcsec FWHM resolution. The remaining $11 \%$ of the image area suffers, at worst, a 0.2 magnitude decrease in limiting magnitude compared to the specification, a difference that was deemed not to be scientifically important on that small of the array. Therefore, it was decided that the marginal return did not justify the high risk and schedule impact of disassembly and re-shimming the CCDs.

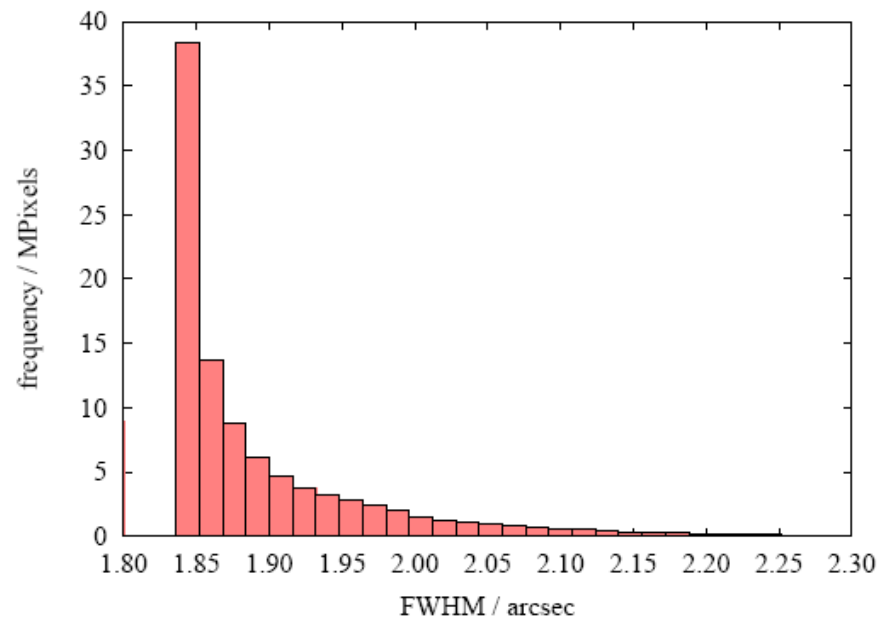

Fig. 11: Distribution of image sizes produced by the measured residual CCD tilt and piston errors in median (1.1 arcsec) seeing in $\mathrm{R}$ band when combined with the image profiles predicted by the optics (predicted by Zemax) at various points on the focal plane.

\section{READOUT ELECTRONICS AND SOFTWARE}

The typical open-shutter exposure time for the PTF survey will be 60 seconds. A requirement was set for readout time to be less than 30 seconds so that observing efficiency will not fall below $66 \%$, while read noise was not to exceed 10 eso that noise would be dominated by shot noise in the sky for the g' and R filters ${ }^{[4]}$.

CFHT wired separate Generation II CCD controller from Astronomical Research Cameras Inc. (ARC) to the six detectors on the upper and lower halves of the mosaic. Although the CCDID-20 CCDs have two outputs, some of the devices have only one functional ${ }^{[2]}$. Since readout must be synchronous, all CCDs must therefore be read through a single output.

The readout time at CFHT was 58 seconds, with a pixel time of approximately $7 \mu$ s, of which only $1+1 \mu$ s was allocated to signal sampling, with read noise typically better than $4 \mathrm{e}^{[2]}$. By shortening the times for CCD reset and serial clocking and overlapping these activities we were able to reduce the pixel time to $3.6 \mu$ s without any noise penalty, since the signal sampling time (and thus noise bandwidth) was unchanged. We have yet to closely examine the degradation of the CTE (for a subset for the CCDs) as serial shift time is reduced, as reported by $\mathrm{CFHT}^{[2]}$, but we hope that by overlapping the serial shift with the entire pixel we will be able to minimize this effect.

The original computer and Astronomical Research Camera's GenII PCI interface card driver supplied by CFHT are now a decade old and are unable to support the data transfer rate needed to reduce the readout time below 45 seconds. Our first step was to upgrade to a newer computer (Pentium 4, $3 \mathrm{GHz}$ ) and a recent version of Linux (2.6 kernel). We also have replaced both the PCI and Timing boards with GenIII boards, which required the assembler code for the waveforms be ported to the DSP in the new Timing board. 
We thank Bob Leach and his staff at ARC for his assistance in making these changes to the DSP firmware and for upgrading the PCI driver and providing test routines to allow two PCI cards to run concurrently on the same bus. Although a single controller and fiber link could read the 12 channels at the $3.44 \mathrm{Mpix} / \mathrm{s}$ rate required for 30 seconds readout time $(68.8 \mathrm{Mbit} / \mathrm{s}$ on the $250 \mathrm{Mbit} / \mathrm{s}$ fiber link), by supporting two fiberlinks and PCI interfaces we avoid having to rebuild the electronics boxes and wiring to the CCDs.

The electronic boxes, if left in their original location (directly attached to the dewar), would have obstructed the incoming beam, interfered with the spiders supporting the focusing hub and would have dissipated excessive power inside the telescope. Therefore, the camera electronics have been moved to the exterior of the closed telescope tube. The cables from the hermetic connectors to the electronics run along the top and bottom of the South spider arm without causing additional beam obstruction. The $\sim 2.4 \mathrm{~m}$ cable length places an additional $80 \mathrm{pF}$ capacitance on the video output but since the CCID-20 package includes a U309 buffer FET with source follower output impedance of approximately $75 \Omega$, the settling time to $0.1 \%(6.9 \tau)$ will be $\sim 50 \mathrm{~ns}$ which will have only moderate impact on read rate. Initial tests with a long cable in the lab have shown that by using proper shielding, RF interference on the extended cables will not cause the $10 \mathrm{e}$ - total readout noise goal to be exceeded.

The new PCI interface is no longer compatible with the original CFHT data acquisition software. Rather than re-write the CFHT interface we instead chose to configure our in-house software package (ArcVIEW) to run the CCD mosaic. ArcVIEW is already in use at Palomar to run a variety of CCDs and IR detectors using current versions of ARC controllers and Linux operating systems.

\section{SUMMARY}

The space and mass limitations at the prime focus of the 48-inch Oschin Schmidt telescope, scientific requirements of the Palomar Transient Factory project (particularly the faster exposure cadence), and the age of the electronics and host operating system, required extensive modifications to the original CFH12K camera. Its most valuable part, the focal plane assembly, has been retained together with the front section of the dewar, the electrical wiring and substantial portions of the CCD controller and its power supplies.

A new closed cycle cooling system replaces the original liquid nitrogen reservoir. New getters, both activated carbon and zeolite, have been installed and some leaks through connectors fixed. Thermal control will be performed by commercial electronics from Lakeshore Cryogenics. New support interface with tip-tilt adjustment will be provided on the back surface instead of the front flange. A field flattener, relocated much closer to the CCDs, has replaced the original window. A compact two-filter changer has been designed having acceptable beam obstruction. A custom compact shutter with precise slower motion was purchased to obtain longer operating life while minimizing beam obstruction. Readout time has been reduced with minimal noise penalty and controller electronics and software were updated to support the faster read rate and interface to the PTF queue scheduler ${ }^{[4]}$. Careful attention has been given to optimizing delivered image quality to the extent possible within budget and performance constraints imposed by the telescope.

The result is a camera system which will run reliably and continuously, unattended, with minimal maintenance. It will produce high quality data while maximizing survey rate at fast cadences. Commissioning is scheduled for the last quarter of 2008.

\section{REFERENCES}

[1] Cenko, B. et al, "The Automated Palomar 60-Inch Telescope", PASP, 118, 1396 (2006)

[2] Cuillandre, J., Luppino, G. A., Starr, B. M., and Isani, S., "Performance of the CFH12K. A 12K by 8K CCD mosaic camera for the CFHT prime focus", Astronomical Telescopes and Instrumentation 2000, Proc. SPIE 4008, 1010 (2000)

[3] Palomar Transient Factory Science Requirements Document (2008)

[4] Palomar Transient Factory Functional Requirements Document (2008)

[5] C. J. Walsh, A. J. Leistner, J. Seckold, B. F. Oreb, and D. I. Farrant, "Fabrication and Measurement of Optics for the Laser Interferometer Gravitational Wave Observatory," Appl. Opt. 38, 2870-2879 (1999) 